\title{
Erratum to: Periosteal mesenchymal chondrosarcoma of the tibia with multifocal bone metastases: a case report
}

\author{
Rajendra Kumar $^{1}$ - Cihan Duran ${ }^{1}$ Behrang Amini ${ }^{1}$ - Dejka M. Araujo ${ }^{2}$. \\ Wei-Lien Wang ${ }^{3}$
}

Erratum to: Skeletal Radiol

DOI 10.1007/s00256-017-2626-5

The original version of this paper unfortunately mis-spelled the name of Wei-Lien Wang and is now corrected in the authorgroup of this article.

The online version of the original article can be found at http://dx.doi.org/ 10.1007/s00256-017-2626-5

Rajendra Kumar

rajkumar@mdanderson.org

1 Department of Diagnostic Imaging, The University of Texas M.D., Anderson Cancer Center, Unit 1475, 1515 Holcombe Blvd, Unit 1475, Houston, TX 77030, USA

2 Department of Medical Oncology, The University of Texas M.D., Anderson Cancer Center, Unit 1475, 1515 Holcombe Blvd, Unit 1475, Houston, TX 77030, USA

3 Department of Pathology, The University of Texas M.D. Anderson Cancer Center, Unit 1475, 1515 Holcombe Blvd, Unit 1475,

Houston, TX 77030, USA 